\title{
Ein Fall von Skew Deviation und Downbeat- Nystagmus infolge von Lithium-Toxizität
}

\author{
Hyunkyu Hong ${ }^{a}$ In Jeong Lyua ${ }^{a}$ b \\ aDepartment of Ophthalmology, Nowon Eulji Medical Center, Eulji University School of Medicine, Seoul, Republik Korea; \\ ${ }^{b}$ Department of Ophthalmology, Korea Cancer Center Hospital, Korea Institute of Radiological and Medical Sciences, Seoul, Republik Korea
}

\section{Schlüsselwörter}

Lithium · Nystagmus · Skew Deviation

\begin{abstract}
Zusammenfassung
Hintergrund: Lithium-Salze finden häufige Anwendung als Prophylaxe oder Behandlung bei bipolaren Störungen und haben zahlreiche Nebenwirkungen. Allerdings gibt es keine Berichte über Skew Deviation und Downbeat-Nystagmus im Zusammenhang mit Lithium. Wir beschreiben hier den ersten Fall von Lithium-induzierter Skew Deviation und Downbeat-Nystagmus.
\end{abstract}

Vorstellung des Falls: Eine 39 Jahre alte Frau stellte sich mit seit 1-2 Monaten bestehender intermittierender vertikaler Diplopie und Schwindelgefühl vor. Die ophthalmologische Untersuchung ergab einen Downbeat-Nystagmus und eine Hypertropie von 6 Prismen-Dioptrien rechts. Die Fundoskopie zeigte eine leichte Inzyklotorsion des rechten Auges. Allerdings waren Duktionen und Versionen im Normbereich. Die übrigen neurologischen Untersuchungen waren ebenfalls ohne Befund. Die Frau hatte eine bipolare Störung, die seit 6 Jahren mit 600-900 mg Lithium pro Tag behandelt wurde. Zwei Monate bevor sie sich zum ersten Mal bei uns vorstellte, war die Lithium-Tagesdosis auf 1200 mg erhöht worden. Wir überwiesen die Patientin an einen Psychiater. Trotz eines Lithium-Serumspiegels im normalen therapeutischen Bereich wurde die Lithium-Tagesdosis der Patientin zunächst auf 600 mg reduziert und Lithium danach abgesetzt. Sechs Tage nach Absetzen von Lithium waren der Downbeat-Nystagmus und die rechtsseitige Hypertropie der Patientin vollständig abgeklungen und die Symptome traten über den Zeitraum von $1 \mathrm{Jahr}$ nicht wieder auf. Schlussfolgerung: Auch wenn die Serumspiegel im normalen therapeutischen Bereich liegen, können als Nebenwirkung von Lithium Downbeat-Nystagmus und Skew Deviation auftreten. Eine Dehydratation kann zur Neurotoxizität von Lithium beitragen.

(c) 2019 The Author(s)

\section{Hintergrund}

Lithium-Salze finden häufige Anwendung als Prophylaxe und Behandlung bei bipolaren Störungen. Lithium hat zahlreiche $\mathrm{Ne}-$ benwirkungen, die auch bei Lithium-Serumspiegeln im normalen therapeutischen Bereich auftreten können [1]. Als neurotoxische Nebenwirkung von Lithium kann ein Downbeat-Nystagmus auftreten [2]. Allerdings gibt es keine Berichte über Skew Deviation und Downbeat-Nystagmus im Zusammenhang mit Lithium. Dies ist der erste Bericht über einen potenziellen Fall von Lithium-Toxizität, bei dem es zusätzlich zu einem Downbeat-Nystagmus zu Skew Deviation kam.

\section{Fallvorstellung}

Die bestkorrigierte Sehschärfe betrug im rechten Auge 20/32 und im linken Auge 20/40. Bei der Spaltlampenuntersuchung waren in beiden Augen Trübungen im Bereich der Linsenrinde und der hinteren Linsenkapsel zu erkennen. Während die Duktionen und Versionen normal waren, hatte die Patientin eine Hypertropie von 6 Prismen-Dioptrien (PDPT) rechts und einen DownbeatNystagmus (Abb. 1). Die Hypertropie bestand auch im Kopfneigetest fort. Der Downbeat-Nystagmus wurde bei allen Blickrichtungen beobachtet, war jedoch beim Blick nach oben leicht unterdrückt. Bei der Fundoskopie war eine leichte Inzyklotorsion des 


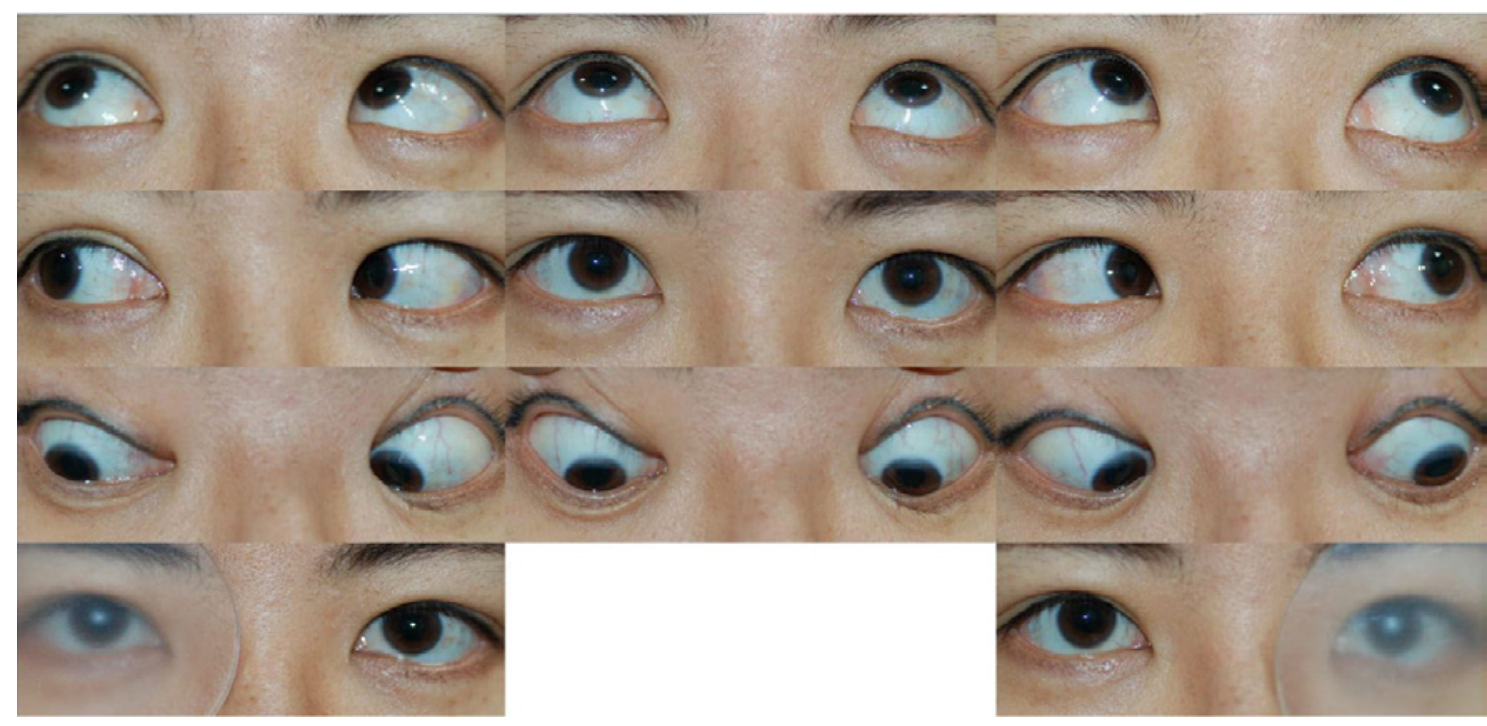

Abb. 1. Fotografien in 9 Blickrichtungen ergaben normale Duktionen und Versionen. Allerdings zeigte die Patientin im alternierenden Prismenabdecktest einen Downbeat-Nystagmus und eine Hypertropie von 6 Prismen-Dioptrien rechts.

rechten Auges zu erkennen (Abb. 2). Alle weiteren neurologischen Untersuchungen ergaben unspezifische Befunde.

Die Patienten litt an einer bipolaren Störung und wurde seit 6 Jahren mit 600-900 mg Lithium pro Tag behandelt. Zwei Monate vor ihrem ersten Besuch bei uns war ihre Lithium-Tagesdosis auf 1200 mg erhöht worden, um ihre bipolare Symptomatik unter Kontrolle zu bringen. Ihre weitere Medikation bestand in Quetiapin $175 \mathrm{mg} /$ Tag, Clonazepam $1 \mathrm{mg} / \mathrm{Tag}$, Valproat $1250 \mathrm{mg} / \mathrm{Tag}$, Lamotrigin $200 \mathrm{mg} / \mathrm{Tag}$, Propranolol $40 \mathrm{mg} / \mathrm{Tag}$, Topiramat $50 \mathrm{mg} / \mathrm{Tag}$ und Ziprasidon $40 \mathrm{mg} /$ Tag. In der Anamnese der Patientin fanden sich kein Alkoholmissbrauch und keine relevanten Virusinfektionen. Eine interessante Beobachtung war, dass sich die Diplopie und Oszillopsie direkt nach Konsum von Sportgetränken sowie nach intravenöser Flüssigkeitszufuhr besserten. Aus diesem Grund wiederholten wir die Augenuntersuchung 20 Minuten, nachdem die Patientin Sportgetränke zu sich genommen hatte, und konnten bestätigen, dass der Nystagmus und die Hypertropie nach Konsum der Sportgetränke nachgelassen hatten.

Weitere geplante Untersuchungen waren eine Magnetresonanztomographie (MRT) des Gehirns, Laboruntersuchungen und ein psychiatrisches Konsil zur Beurteilung der Fragestellung, ob es sich um durch Lithium induzierte Nebenwirkungen handeln könnte. Der Lithium-Spiegel der Patientin betrug $0,8 \mathrm{mEq} / \mathrm{l}$ (therapeutischer Bereich: 0,6-1,0 mEq/l) und es gab keine Hinweise auf eine Elektrolytstörung. Die Serumspiegel von Natrium, Kalium, Calcium und Chlorid waren im Normbereich, ebenso wie der Vitamin-B12-Spiegel.

Im Anschluss an das psychiatrische Konsil wurde die LithiumDosis auf $600 \mathrm{mg}$ reduziert und Lithium dann innerhalb von 1 Woche abgesetzt. Allerdings lehnte die Patientin weitere Untersuchungen, darunter die MRT des Gehirns, ab. Sechs Tage nach Absetzen von Lithium waren der Downbeat-Nystagmus und die rechtsseitige Hypertropie der Patientin vollständig abgeklungen und die Symptome traten über den Zeitraum von 1 Jahr nicht wie- der auf. Die Patientin gab nach Aufklärung ihre schriftliche Einwilligung für die Publikation.

\section{Diskussion und Schlussfolgerungen}

Ein Downbeat-Nystagmus ist eine bekannte mögliche Manifestationsform einer Lithium-Toxizität, während die häufigste Störung der Augenmotorik im Zusammenhang mit Lithium ein horizontaler Blick-evozierter Nystagmus ist [3]. Weitere okuläre Komplikationen wie ein periodisch alternierender Nystagmus, sakkadischer Pursuit, okulogyre Krisen und Opsoklonus wurden ebenfalls auf eine Lithium-Toxizität zurückgeführt [3].

In früheren Studien wurde beschrieben, dass bei der Pathogenese eines Lithium-induzierten Downbeat-Nystagmus Schädigungen des Nucleus prepositus hypoglossi (NPH), des Nucleus vestibularis medialis und des Kleinhirns eine Rolle spielen [3, 4]. Während der genaue Mechanismus dieser Schädigungen nicht bekannt ist, ist meist eine chronische Lithium-Exposition anstelle einer akuten Toxizität die Ursache [3, 5]. Betroffene Patienten nahmen Lithium in Dosen zwischen 500 und $1800 \mathrm{mg}$ pro Tag ein $[3,6,7]$. Ein Downbeat-Nystagmus kann bei therapeutischem LithiumSerumspiegel auftreten und auch nach Absetzen des Arzneimittels persistieren [3, 6-10]. Mit einer Lithium-Toxizität assoziierte Faktoren sind neue Arzneimittel, die die Nierenfunktion beeinflussen (wie Angiotensin-Converting-Enzyme-Hemmer und nicht-steroidale Antirheumatika), Dehydratation, febrile Infekte und Gastroenteritis [11]. Während nach versehentlicher oder vorsätzlicher Überdosierung von Lithium schwere neurologische Symptome auftreten können, können bei chronischer Toxizität okuläre Manifestationen alleine oder zusammen mit leichten neurologischen Symptomen wie Ataxie oder Tremor bestehen [8]. Als begleitend zu einem Downbeat-Nystagmus auftretende neuroophthalmologische Manifestationen wurden verschwommenes Sehen [6] und horizontale Blicklähmung [3, 7] beschrieben. 


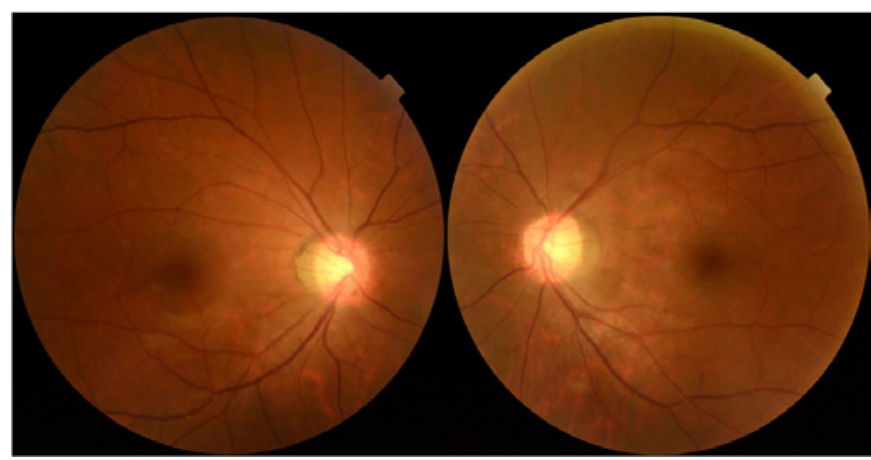

Abb. 2. Fundus-Photographien zeigten eine leichte Inzyklotorsion im rechten Auge und ein normales linkes Auge.

Skew Deviation ist definiert als vertikale Fehlstellung der Augen aufgrund eines asymmetrischen supranukleären Inputs mit Beteiligung von Otolith-okulären Bahnen [12]. Die Störung kann bei verschiedenen Anomalien des vestibulären Systems, Hirnstamms oder Kleinhirns auftreten und mit weiteren neurologischen Symptomen assoziiert sein [13, 14]. In der Vergangenheit wurde bereits über Skew Deviation und Downbeat-Nystagmus bei einem Patienten mit Läsion im NPH, einem zentralen Bestandteil des neuronalen Netzwerkes von vestibulärem System, Kleinhirn und Hirnstamm, berichtet [15]. Allerdings ist der vorliegende unseres Wissens nach der erste Bericht über einen potenziellen Fall von Lithium-Toxizität, bei dem es zusätzlich zu einem Downbeat-Nystagmus zu Skew Deviation kam.

Ein weiteres besonderes Merkmal unseres Falls ist, dass sich die Symptome der Patientin nach intravenöser Flüssigkeitstherapie oder Konsum eines Sportgetränks vorübergehend besserten. Unabhängig von seinem Ursprung ist ein Volumenmangel eine häufige Ursache für eine chronische Lithium-Intoxikation, und Hydrierung hilft, die Lithium-Clearance zu maximieren [11]. In unserem Fall schienen der Konsum von Sportgetränken und eine intravenöse Flüssigkeitstherapie die Symptome vorübergehend unter Kontrolle zu bringen. Darüber hinaus unterstreicht unser Fall, dass ein frühes Erkennen neuroophthalmologischer Symptome bei Patienten, bei denen Verdacht auf eine Lithium-Toxizität besteht, obwohl der Lithium-Spiegel im normalen therapeutischen Bereich liegt, und ein sofortiges konsequentes Absetzen des Arzneimittels, die Chancen erhöhen könnten, irreversible Folgen zu vermeiden $[6,8]$. Die vorliegende Studie weist mehrere Begrenzungen auf. Da die Patientin dies ablehnte, wurde keine MRT des Gehirns angefertigt und der Magnesium-Serumspiegel nicht bestimmt. Allerdings fanden sich in der Anamnese der Patientin kein Alkoholmissbrauch und keine relevanten Virusinfektionen. Die Patientin hatte normale neurologische Befunde und normale Serumspiegel von Vitamin B12 und Valproat sowie Elektrolyten, was die Wahrscheinlichkeit anderer Ursachen für Skew Deviation und Downbeat-Nystagmus verringerte. Weitere mögliche Ätiologien eines Downbeat-Nystagmus sind strukturelle Läsionen im Gehirn und metabolische Störungen wie Hypomagnesiämie, Thiamin-Mangel und Vitamin-B12-Mangel $[16,17]$. Auch eine Lamotrigin-Toxizität wurde als seltene Ursache eines Downbeat-Nystagmus be- schrieben, wobei die Halbwertzeit bei Anwendung zusammen mit Valproat ansteigt [18]. Auch wenn unsere Patientin Lamotrigin einnimmt, ist festzustellen, dass die vertikale Diplopie und der Nystagmus erst nach Erhöhung der Lithium-Tagesdosis auftraten und dass sie nach Absetzen von Lithium vollständig abklangen, ohne dass die sonstige Medikation der Patientin geändert wurde. Dies spricht für die Annahme, dass sowohl die Skew Deviation als auch der Downbeat-Nystagmus in diesem Fall auf die Einnahme von Lithium zurückgeführt werden können.

Als Schlussfolgerung ist festzustellen, dass bei Patienten unter langfristiger Lithium-Therapie auch bei Spiegeln im therapeutischen Bereich Skew Deviation und Downbeat-Nystagmus auftreten können, und dass sich die Symptome nach Absetzen des Arzneimittels bessern können.

\section{Verfügbarkeit der Daten und Materialien}

Die Daten, die die Schlussfolgerungen dieses Artikels unterstützen, sind im Artikel enthalten.

\section{Finanzierung der Studie}

Diese Arbeit wurde durch Fördermittel des Korea Institute of Radiological and Medical Sciences (KIRAMS), finanziert durch das Ministery of Science and ICT (MSIT), Republik Korea (Nr. 50541-2019), unterstützt. Die finanzierenden Stellen waren nicht an Design oder Durchführung dieser Studie beteiligt.

\section{Beiträge der Autoren}

IJL war für das Design der Studie verantwortlich, versorgte die Patientin, analysierte die Daten, schrieb das Manuskript und überarbeitete das Manuskript. HH war an der Erhebung und Analyse der Daten, am Schreiben des Manuskripts und an der Überarbeitung des Manuskripts beteiligt. Alle Autoren haben die finale Fassung des Manuskripts gelesen und freigegeben.

\section{Genehmigung durch die Ethikkommission und Einwilli- gung zur Teilnahme}

Die Studie wurde in Übereinstimmung mit den Prinzipien der Deklaration von Helsinki durchgeführt. Das Institutional Review Board des Nowon Eulji Medical Center (Seoul, Republik Korea) genehmigte die Durchführung dieser Studie.

\section{Zustimmung zur Veröffentlichung}

Von der Patientin wurde eine schriftliche Einwilligung nach Aufklärung für die Veröffentlichung des vorliegenden Fallberichts eingeholt. Ein Exemplar der schriftlichen Einverständniserklärung ist zur Überprüfung durch das Redaktionsteam des BMC Ophthalmology verfügbar.

\section{Disclosure Statement}

Die Autoren erklären, dass keine Interessenskonflikte bestehen.

\section{Lizenzangabe}

Hong H, Lyu IJ: XYZ. A case of skew deviation and downbeat Nystagmus induced by Lithium. BMC Ophthalmology 2019;19:257 (DOI: 10.1186/ s12886-019-1270-3). ${ }^{\circ} 2019$ The Author(s). (Übersetzung; Publisher's Note gekürzt), lizensiert unter CC BY 4.0 (https://creativecommons.org/licenses/by/4.0/deed.de).

\section{Literatur}

Die Literatur ist unter www.karger.com/doi/10.1159/000512225 abrufbar. 